\title{
Screening and functional analysis of glioma-related genes induced by candoxin
}

\author{
LEI QIU $^{1 *}$, XIANG ZHANG ${ }^{2 *}$ and ZUOQUAN CHEN ${ }^{2}$ \\ ${ }^{1}$ Department of Neurosurgery, Zibo Central Hospital, Zibo, Shandong 255036; ${ }^{2}$ Department of Neurosurgery, \\ The Tenth People's Hospital Affiliated to Shanghai Tongji University, Shanghai 200072, P.R. China
}

Received October 30, 2013; Accepted May 9, 2014

DOI: $10.3892 / \mathrm{mmr} .2014 .2311$

\begin{abstract}
This study aimed to identify time-specific and common differential genes (CDGs) expressed in glioma cells following exposure to candoxin at three different time-points. Gene expression data from candoxin-treated human glioma (Hs 683) cells were downloaded from the Gene Expression Omnibus database (accession number, GSE1682), from a 12-time-point set of samples. Differentially expressed genes were screened between control and candoxin-treated samples at different time-points, and three time-specific gene sets and CDGs were identified. All genes were subject to pathway enrichment analysis to gain further insight into gene function. CDGs were clustered based upon their original expression between 0 and $48 \mathrm{~h}$, and, from this subset, feature genes were selected. Small molecules associated with candoxin were identified by comparing the expression pattern of the most valid candidate genes with that of differential genes exposed to small molecules in the Connectivity Map. From the 12-, 24- and 48-h time-points, 746, 265 and 539 differentially expressed genes were identified, respectively. A total of 129 genes were differentially expressed and significantly enriched in focal adhesion and gap junction pathways. From these, 11 feature genes and one marker gene (EPS8L1) were identified. Four small molecules that were most relevant to candoxin action were identified. In conclusion, it is hypothesized that candoxin stimulation can cause glial inflammation through mutations in cell adhesion activity. The EPS8L1 gene may be a valid marker for glioma diagnosis, and the four small molecules identified may be relevant for future drug design.
\end{abstract}

Correspondence to: Dr Zuoquan Chen, Department of Neurosurgery, The Tenth People's Hospital Affiliated to Shanghai Tongji University, 301 Middle Yanchang Road, Zhabei, Shanghai 200072, P.R. China

E-mail: chenzuoquanczq@hotmail.com

${ }^{*}$ Contributed equally

Key words: glial inflammation, differentially expressed gene, pathway analysis, feature gene, small molecules

\section{Introduction}

The occurrence of brain tumors has steadily increased over recent years, with glioma having the highest incidence and mortality rate amongst all the intracranial primary tumors (1). These tumors are highly invasive and aggressive with a mean survival time of 15-18 months from diagnosis to death (2).

Various genes have been reported to be associated with tumor formation. $\beta$-catenin is a key gene involved in the regulation of gene expression during tumorigenesis (3), and the RET protooncogene encoding a receptor tyrosine kinase is important in the development of endocrine neoplasia syndromes and familial medullary thyroid carcinoma (4). Furthermore, TNC expression correlates with malignancy in glioblastoma (5), and common variants in SOD2, SOD3 and CAT genes may influence brain tumor risk (6). In addition, hTERTC27, a constructed polypeptide, exerts its effects through complex mechanisms, involving genes associated with cell adhesion. (7).

Data from multiple levels have been utilized to explore the mechanism underlying glioma formation. Aberrant epigenetic regulation of gene expression contributes to tumor initiation and progression (8), and post-translational modifications on histone tails are believed to affect a variety of chromatin processes in the development of malignant brain tumors (9). Digital karyotyping and single nucleotide polymorphism arrays have been utilized to screen glioblastoma tumor cells for changes in copy-number (10). The most frequently amplified region contains the genes EGFR and SEC61 $\gamma$, which are important in glioblastoma multiforme cell survival (10).

It has recently been reported that the molecular pathways of invasion and inflammation are associated with cancer (11). Tumors are frequently observed to originate at sites of chronic inflammation, and inflammatory cells may be observed in tumors. Additionally, it has been estimated that $15-20 \%$ of all mortalities from cancer worldwide are linked to underlying infections and inflammatory responses (12). Non-steroidal anti-inflammatory drugs reduce the risk of developing certain cancers, including colon and breast cancer, and reduce the likelihood of mortality (13). These observations indicate that inflammatory cells, including tumor-associated macrophages, can destroy tumor cells, in addition to normal tissue cells (14). Signaling pathways that are associated with 
inflammation act downstream of oncogenic mutations, such as mutations in the genes encoding RAS, MYC and RET (13).

Candoxin, is a three-finger neurotoxin that can inhibit postsynaptic neuromuscular and neuronal $\alpha 7$ nicotinic acetylcholine receptors and delay apoptosis of glial cells. Exposure to candoxin has been shown to trigger glial inflammation and neurodegenerative diseases (15). If stimulated by inflammation, original cells at the residual foci of infection can develop into tumor-forming cells through chronic proliferation. Similarly, glial inflammation can develop into glioma if not effectively diagnosed and treated.

In the present study, a gene expression profile of glioma cells exposed to candoxin at different time-points was used to screen for time-specific differentially expressed genes in order to perform functional and pathway analysis. Using data from the Connectivity Map, expression patterns were compared to identify other drugs that exhibited similarities with candoxin. This approach may guide drug design for the treatment of glial inflammation (16).

\section{Materials and methods}

Data source. Microarray data were downloaded from the Gene Expression Omnibus (http://www.ncbi.nlm.nih.gov/geo/; accession number, GSE1682) (15). In this time-course dataset, the global gene expression of human Hs 683 glioma cells was detected 12, 24 and $48 \mathrm{~h}$ after candoxin exposure. All 12 samples included three samples for each time-point and three untreated control cells. The microarray platform used was GPL96 (HG-U133A).

The Connectivity Map database (http://www.broad.mit. edu/Connectivity Map/) consists of >7,000 gene expression profiles following treatment with 1,309 small molecules (17). These expression profiles represent $\sim 6,000$ cases, each of which comprises a treatment and vehicle pairing. By comparing the similarity of expression pattern of genes in pathways and the genes perturbed in the Connectivity Map cases, a list of molecules with an effect on the input genes was identified.

Screening of differential genes. Missed values were inferred (18) and then the data were normalized (19). Up-and downregulated genes were identified between cancer samples at each time-point and control using the $\mathrm{R}$ package limma (Affymetrix, Santa Clara, CA, USA; http://www.affymetrix.com/analysis/) (20). According to the fold-difference method (21), if the fold difference was $>1$, the gene was regarded as a differential gene in the study. The Benjamini-Hochberg false discovery rate (22) was performed for multiple testing correction $(\mathrm{P}<0.05)$. Finally, three sets of time-course specific differential genes (12, 24 and $48 \mathrm{~h}$ ) and a set of common differential genes (CDGs) at the three time-points were identified.

Detection of significant pathways. The Database for Annotation, Visualization and Integrated Discovery annotation system (DAVID Bioinformatics Resources 6.7; http://david.abcc.ncifcrf.gov/home.jsp) was used to interpret the gene list from the microarray data (23) and an Expression Analysis Systematic Explorer score (a modified Fisher Exact)
Table I. Calculation of EASE score in DAVID.

\begin{tabular}{lcc}
\hline & User genes & Genome \\
\hline In pathway & $\mathrm{a}-1$ & $\mathrm{~b}$ \\
Not in pathway & $\mathrm{c}$ & $\mathrm{d}$ \\
\hline
\end{tabular}

$\mathrm{n}=(\mathrm{a}+\mathrm{b}+\mathrm{c}+\mathrm{d})$ : $\mathrm{a}$, total number of user genes annotated in a pathway; $b$, number of genes annotated in this pathway; c, number of user genes not annotated in this pathway; $d$, number of background genes not annotated in this pathway. EASE, Expression Analysis Systemic Explorer; DAVID, Database for Annotation, Visualization and Integrated Discovery.

was adopted to measure the degree of gene-enrichment in the pathways (Table I). The equation used is shown below:

$$
P=\frac{\left(\begin{array}{c}
a+b \\
a
\end{array}\right)\left(\begin{array}{c}
c+d \\
c
\end{array}\right)}{\left(\begin{array}{c}
n \\
a+c
\end{array}\right)}=\frac{(a+b) !(c+d) !(a+c) !(b+d) !}{a ! b ! c ! d !}
$$

In this formula, where $n=(a+b+c+d)$, a was the total number of user genes annotated in a pathway; $b$ was the number of genes annotated in this pathway; c was the number of user genes not annotated in this pathway and d was the number of background genes not annotated in this pathway. The symbol '! represents factorial function, which multiplies a series of descending natural numbers When $\mathrm{P}<0.05$ it was hypothesized that the user gene lists were statistically significantly associated (enriched) in this pathway.

Detecting feature genes in CDGs. Feature genes were determined from the CDGs at the three time-points. By using the open source software Cluster (Cluster 3.0; http://bonsai.hgc. jp/ mdehoon/software/cluster/index.html) (24), the expression values of CDGs in all 12 samples were clustered, and the genes whose expression values altered with flip features were defined as feature genes for glioma.

Detecting drugs with similarities to candoxin based upon gene expression pattern comparison. The connectivity score (S) was used to detect drugs with similarity to candoxin. For the up- and downregulated genes, KS up and down scores $\left(\mathrm{KS}_{\mathrm{up}}\right.$ and $\mathrm{KS}_{\mathrm{down}}$, respectively) were calculated, as follows:

$$
\begin{gathered}
a=\max _{j=1}^{t}\left[\frac{j}{t}-\frac{V(j)}{N}\right], \quad b=\max _{j=1}^{t}\left[\frac{V(j)}{N}-\frac{j-1}{t}\right] \\
K S_{\text {up } / \text { down }}=\left\{\begin{array}{l}
a,(a>b) \\
-b,(b>a)
\end{array}\right.
\end{gathered}
$$

In this formula, $\mathrm{t}$ was the number of genes in either the up- or downregulated gene group, $\mathrm{N}$ was the total number genes in the array, $\mathrm{j}$ denoted the jth gene in the rank-ordered up- or downregulated groups according to the extent of differential expression and $\mathrm{V}(\mathrm{j})$ denoted the position of the jth gene in the rank-ordered whole gene list (also ranked according to 


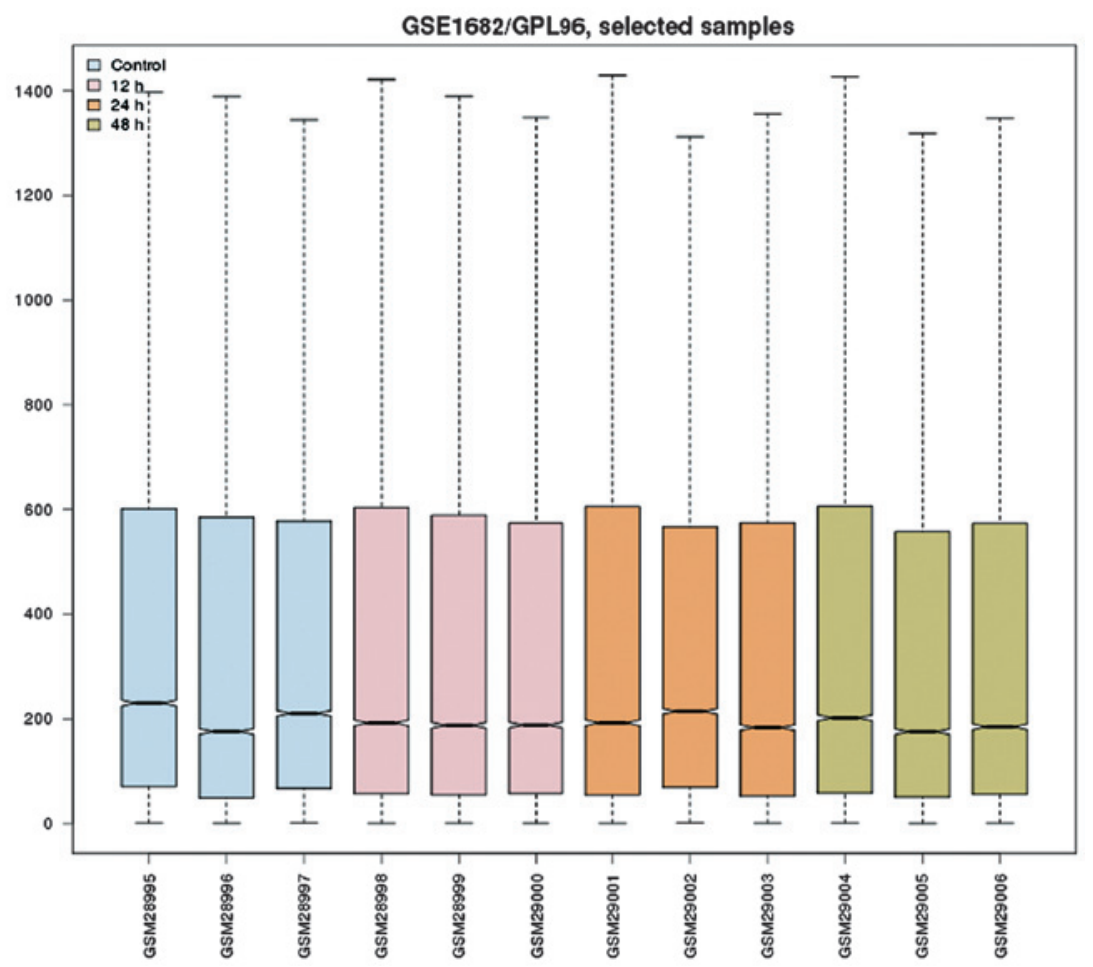

Figure 1. Box-plot of normalized gene expression for each sample. The blue boxes represent three normal samples, and the pink, orange and green boxes represent gene expression of samples exposed to candoxin after 12, 24 and $48 \mathrm{~h}$, respectively. The normalization method used was based on the median. All the medians were approximately equivalent.

the extent of differential expression). The connectivity score $\mathrm{S}$ was set to zero where $\mathrm{KS}_{\text {up }}$ and $\mathrm{KS}_{\text {down }}$ had the same sign; otherwise, $\mathrm{S}$ was set as $\mathrm{KS}_{\mathrm{up}}-\mathrm{KS}_{\text {down }}$.

\section{Results}

Time-point-specific differential genes. Following data pre-processing, high standardization among samples was observed (Fig. 1). Time-point-specific differential genes were detected, with 1,335, 645 and 1,087 differentially expressed genes at the 12, 24, and $48 \mathrm{~h}$ time-points respectively. The data comprised the gene symbol of differential genes and the expression difference of the genes between the glioma cells and controls.

The time-specific differential genes were further analyzed. Numerous genes were differentially expressed at two time-points, while other genes were differentially expressed at all three time-points (Fig. 2). A total of 129 genes were differentially expressed at all three time-points. Finally, 746, 265 and 539 time-point-specific differential genes at the 12-, 24- and 48-h time-points, respectively, were obtained.

Pathway annotation of differential genes. Time-specific differential genes were annotated by Kyoto Encyclopedia of Genes and Genomes pathway analysis. The CDGs were enriched in 'gap junction' function. All the enriched pathways are shown in Table II.

Through pathway enrichment analysis, it was found that all the three time-point-specific genes were enriched in focal adhesion pathways. This indicated that at different time-points, glioma cells exposed to candoxin had time-specific differen-

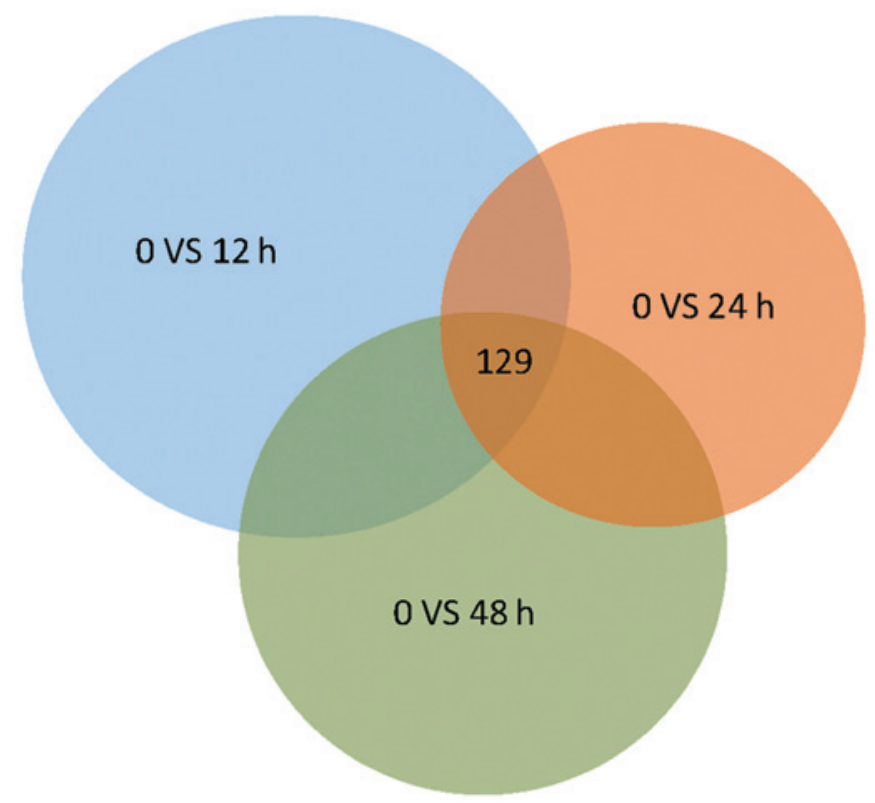

Figure 2. Differential genes identified at three time-points. Blue, orange and green circles represent differential genes at 1,24 and $48 \mathrm{~h}$, compared with the control sample. A total of 129 common differential genes were shared by the three time-points.

tial genes. However, these distinct genes were enriched in the same pathway.

Residual foci of normal cells can develop into tumors following inflammation, which can cause chronic cell proliferation (12). Exposure to candoxin may induce abnormal behavior in focal adhesion and lead to chronic cell prolifera- 
Table II. Significant pathways enriched in the differential gene sets.

A, 0 vs. $12 \mathrm{~h}$

\begin{tabular}{llr}
\hline KEGG ID & \multicolumn{1}{c}{ Function } & P-value \\
\hline hsa04510 & Focal adhesion & $5.61 \times 10^{-6}$ \\
hsa04512 & ECM-receptor interaction & $1.07 \times 10^{-5}$ \\
\hline
\end{tabular}

B, 0 vs. 24 h

\begin{tabular}{lll}
\hline KEGG ID & \multicolumn{1}{c}{ Function } & P-value \\
\hline hsa05416 & Viral myocarditis & $9.37 \times 10^{-4}$ \\
hsa04510 & Focal adhesion & 0.0002936 \\
hsa05200 & Pathways in cancer & 0.014194 \\
\hline
\end{tabular}

C, 0 vs. $48 \mathrm{~h}$

\begin{tabular}{lll}
\hline KEGG ID & \multicolumn{1}{c}{ Function } & P-value \\
\hline hsa04144 & Endocytosis & 0.036075 \\
hsa04510 & Focal adhesion & 0.005635 \\
hsa04010 & MAPK signaling pathway & 0.010646 \\
\hline
\end{tabular}

$\mathrm{D}$, Common differential genes

\begin{tabular}{lll}
\hline KEGG ID & \multicolumn{1}{c}{ Function } & P-value \\
\hline hsa04540 & Gap junction & 0.001711 \\
hsa00230 & Purine metabolism & 0.006882 \\
hsa04020 & Calcium signaling pathway & 0.046127 \\
\hline
\end{tabular}

KEGG, Kyoto Encyclopedia of Genes and Genomes; ECM, extracellular matrix; MAPK, mitogen-activated protein kinase.

tion and consequently to glioma. All time-point-specific genes and CDGs were significantly enriched in cell adhesion-related pathways. It is speculated that, following exposure to candoxin, changes occur in cell adhesion, which results in glial inflammation.

Feature genes in CDGs. Following the identification of differential genes at three time-points, 129 CDGs were obtained. In order to select the most valid candidates, known as feature genes, expression fold changes were utilized to perform the subsequent analyses. CDGs were clustered at three time-points (Fig. 3). From this, it was identified that the majority of the CDGs were downregulated and 118 CDGs were continuously up- or downregulated at the three time-points. The remaining 11 CDGs exhibited different expression patterns at the different time-points. These 11 genes, which are marked on Fig. 3 with a red frame, included CXorf57, SGCD, CRHR1, EPS8L1, DRD2, ICOSLG, SLC39A8, PDGFRA, PADI4, RARA and TAF6L.

The original expression of these 11 genes was observed at three time-points. EPS8L1 was the most significantly upregulated gene at $24 \mathrm{~h}$ and was also upregulated at 12 and $48 \mathrm{~h}$
Table III. Small molecules with similarity score $>0.9$.

\begin{tabular}{lr}
\hline Small molecule & Score \\
\hline Halofantrine & -0.969 \\
Risperidone & -0.906 \\
Menadione & 0.909 \\
Verteporfin & 0.948
\end{tabular}

(Fig. 4). Thus, it was speculated that EPS8L1 may be a marker for glial inflammation, and its overexpression at $24 \mathrm{~h}$ could direct a diagnosis of glial inflammation.

Drugs with similarity to candoxin. Small molecules that exhibited similarities to candoxin were detected through comparing the expression pattern of the 11 CDGs with that of the genes perturbed by small molecules stored in the Connectivity Map. Where the absolute $\mathrm{S}$ score was $>0.9$, the small molecule was screened as a candoxin-related drug. As a result, four drugs, halofantrine, risperidone, menadione and verteporfin, were 


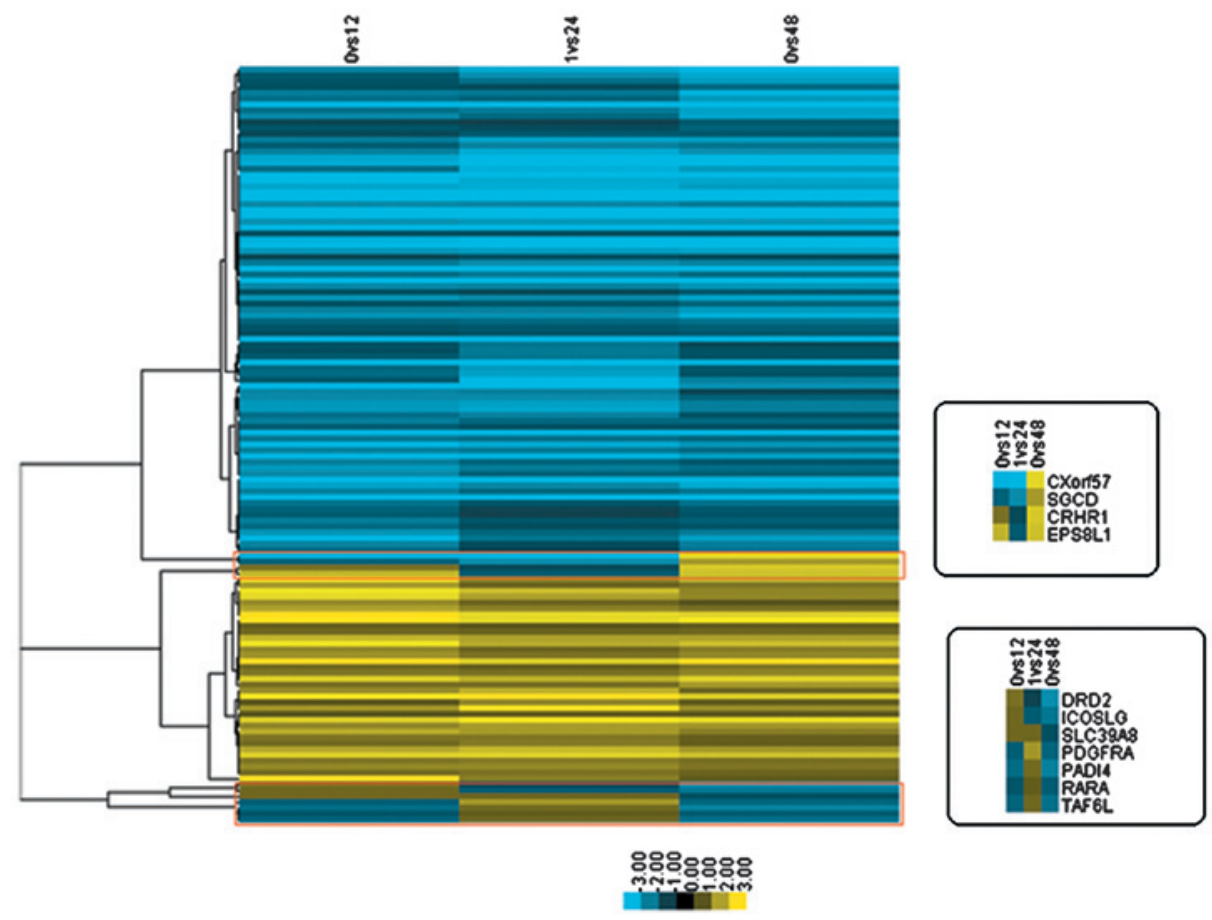

Figure 3. Dendrogram of $\log F C$ for 129 common differential genes. The blue grid represents downregulation, whereas the yellow grid represents upregulation. The darker the blue or yellow color, the greater the extent of gene downregulation or upregulation, respectively. The additional boxes indicate the 11 genes whose expression markedly changed at the three time-points.

identified. Halofantrine and risperidone were positively associated with candoxin, whilst menadione and verteporfin were negatively associated with candoxin (Table III).

\section{Discussion}

An increasing number of approaches are currently used to treat glioma. Further understanding of the mechanisms behind glioma generation and progression is required to provide insight into targeted therapy. The presented study was based on microarray data of glial inflammation, elaborating the mechanism underlying glioma from the perspective of cancer-related inflammation. The results first identified several CDGs at three time-points, which were predicted to be associated with cell adhesion pathways. Focal adhesion kinase (FAK), a target of miR-7, regulates glioblastoma cell invasion. FAK signaling plays a critical role in the production of matrix metallopeptidases (MMPs) such as MMP-2 and MMP-9, and subsequently activates tumor invasion (26). A close association between Connexin 43 and the actin cytoskeleton has been confirmed by preloading assay and further reinforced by the co-staining of full-length Connexin 43 with the actin-rich protrusions in migrating LN18 Connexin 43 cells. The carboxy-terminal tail of Connexin 43 facilitates glioma migration (27). Additionally, cell-cell communication is impaired via gap junctions in glioma cells, which may be due to phosphorylation of serine or tyrosine residues in the C-terminal domain of Connexin 43 (28).

Eleven feature genes were identified that were differentially expressed at three time-points (CXorf57, SGCD, CRHR1, EPS8L1, DRD2, ICOSLG, SLC39A8, PDGFRA, PADI4, RARA and TAF6L). DRD2 has been reported to be expressed in rat glioma (29), which is consistent with the

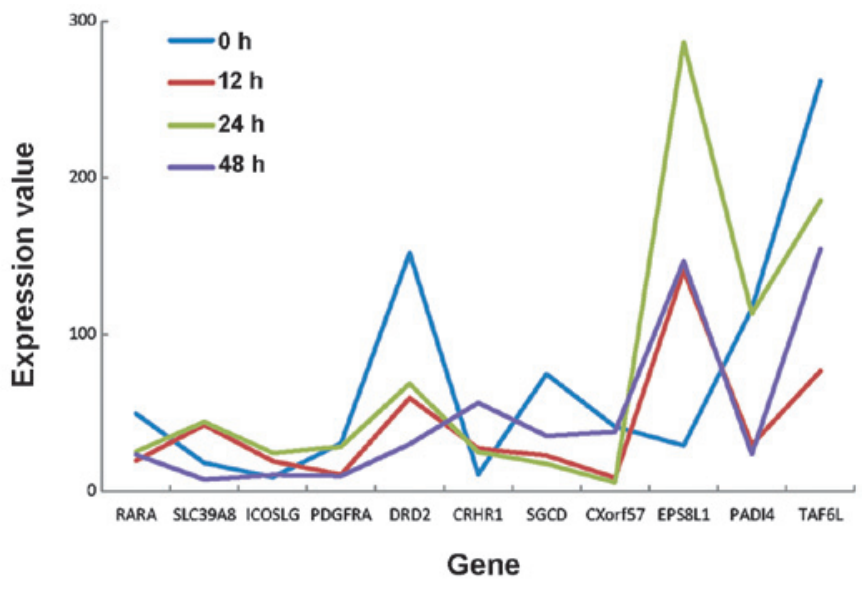

Figure 4. Original gene expression data for 11 common differential genes whose expression markedly changed at the three time-points. Blue, red, green and purple polylines represent gene expression at $0,12,24$ and $48 \mathrm{~h}$, respectively.

findings of the present study. Overexpression of PDGFRA has been identified in one case of recurrent and three cases of primary glioblastoma, and acts as a driver of tumor growth and invasion (30-32). Inhibiting PDGFRA stimulation by knockdown of endogenous Dock180 markedly impairs basal and PDGFA-stimulated Rac1 activities and in vitro cell migration of LN443 and LN444 cells (33). Thus, glioma progression may be associated with focal adhesion (34).

Four small molecules were identified in the present study: Risperidone, menadione, halofantrine and verteporfin. HSP70 may play a significant role in the pathophysiology of schizophrenia; risperidone can decrease HSP70 expression in MK-801-treated rat C6 glioma cells (26) and is able 
to interfere with C6 glioma cell adhesion without toxic effects (27). 2,3-Epoxide substitution significantly potentiates the apoptotic effects of menadione through stimulating reactive oxygen species production, which may be useful in the chemotherapy of glioblastoma cells (28). DNA damage (single-strand breaks) has been associated with the cytotoxic effects of menadione (35).

It is of particular significance to investigate the pathogenesis of glioma and to design targeted therapeutic methods for glioma treatment. Treatment of glial inflammation is additionally important, since it can prevent the formation of glioma from inflammation. Tumor formation is often initialized by the chronic proliferation of inflammatory cells and can significantly affect patients' health. Glial inflammation is less harmful and more feasible to treat than glioma. Thus, timely diagnosis and effective treatment of inflammation should be a priority in order to prevent glioma formation. In this way tumor cells may be destroyed prior to development and the formation of glioma may be prevented. Effective therapeutic strategies are likely to emerge as the understanding of tumor formation is enhanced.

\section{References}

1. Okamoto Y, Di Patre PL, Burkhard C, et al: Population-based study on incidence, survival rates, and genetic alterations of low-grade diffuse astrocytomas and oligodendrogliomas. Acta Neuropathol 108: 49-56, 2004.

2. Castro MG, Candolfi M, Kroeger K, et al: Gene therapy and targeted toxins for glioma. Curr Gene Ther 11: 155-180, 2011.

3. Canal F and Perret C: PKM2: a new player in the $\beta$-catenin game. Future Oncol 8: 395-398, 2012

4. Wells SA Jr and Santoro M: Targeting the RET pathway in thyroid cancer. Clin Cancer Res 15: 7119-7123, 2009.

5. Sivasankaran B, Degen M, Ghaffari A, et al: Tenascin-C is a novel RBPJkappa-induced target gene for Notch signaling in gliomas. Cancer Res 69: 458-465, 2009.

6. Rajaraman P, Hutchinson A, Rothman N, et al: Oxidative response gene polymorphisms and risk of adult brain tumors. Neuro Oncol 10: 709-715, 2008.

7. Ng SS, Gao Y, Chau DH, et al: A novel glioblastoma cancer gene therapy using AAV-mediated long-term expression of human TERT C-terminal polypeptide. Cancer Gene Ther 14: 561-572, 2007.

8. Rahman R, Osteso-Ibanez T, Hirst RA, et al: Histone deacetylase inhibition attenuates cell growth with associated telomerase inhibition in high-grade childhood brain tumor cells. Mol Cancer Ther 9: 2568-2581, 2010.

9. Bonasio R, Lecona E and Reinberg D: MBT domain proteins in development and disease. Semin Cell Dev Biol 21: 221-230, 2010

10. Lu Z, Zhou L, Killela P, et al: Glioblastoma proto-oncogene SEC61gamma is required for tumor cell survival and response to endoplasmic reticulum stress. Cancer Res 69: 9105-9111, 2009.

11. Dikshit B, Irshad K, Madan E et al: FAT1 acts as an upstream regulator of oncogenic and inflammatory pathways, via PDCD4, in glioma cells. Oncogene 32: 3798-3808, 2013.

12. Balkwill F and Mantovani A: Inflammation and cancer: back to Virchow? Lancet 357: 539-545, 2001.

13. Mantovani A, Allavena P, Sica A and Balkwill F: Cancer-related inflammation. Nature 454: 436-444, 2008.

14. Mantovani A, Bottazzi B, Colotta F, Sozzani S, and Ruco L: The origin and function of tumor-associated macrophages. Immunol Today 13: 265-270, 1992.
15. Pachiappan A, Thwin MM, Manikandan $J$ and Gopalakrishnakone P: Glial inflammation and neurodegeneration induced by candoxin, a novel neurotoxin from Bungarus candidus venom: global gene expression analysis using microarray. Toxicon 46: 883-899, 2005.

16. Wen Z, Wang Z, Wang S, et al: Discovery of molecular mechanisms of traditional Chinese medicinal formula Si-Wu-Tang using gene expression microarray and connectivity map. PLoS One 6: e18278, 2011.

17. Lamb J, Crawford ED, Peck D, et al: The Connectivity Map: using gene-expression signatures to connect small molecules, genes, and disease. Science 313: 1929-1935, 2006.

18. Troyanskaya O, Cantor M, Sherlock G, et al: Missing value estimation methods for DNA microarrays. Bioinformatics 17: 520-525, 2001

19. Fujita A, Sato JR, Rodrigues Lde O, Ferreira CE and Sogayar MC: Evaluating different methods of microarray data normalization. BMC Bioinformatics 7: 469, 2006.

20. Smyth GK: limma: Linear Models for Microarray Data. In: Bioinformatics and Computational Biology Solutions Using R and Bioconductor. Gentleman R, Carey VJ, Huber W, Irizarry RA and Dudoit S (eds). Springer, New York, NY, pp397-420, 2005.

21. Butte AJ, Ye J, Häring HU, Stumvoll M, White MF and Kohane IS: Determining significant fold differences in gene expression analysis. Pac Symp Biocomput: 6-17, 2001.

22. Benjamini Y and Hochberg Y: Controlling the false discovery rate: a practical and powerful approach to multiple testing. J R Statist Soc B 57: 289-300, 1995.

23. Huang Da W, Sherman BT and Lempicki RA: Systematic and integrative analysis of large gene lists using DAVID bioinformatics resources. Nat Protoc 4: 44-57, 2009.

24. de Hoon MJ, Imoto S, Nolan J and Miyano S: Open source clustering software. Bioinformatics 20: 1453-1454, 2004.

25. Nagano M, Hoshino D, Koshikawa N, et al: Turnover of focal adhesions and cancer cell migration. Int J Cell Biol 2012: 3106-3116, 2012.

26. Roh K, Roh S, Yang BH, et al: Effects of haloperidol and risperidone on the expression of heat shock protein 70 in MK-801-treated rat C6 glioma cells. Prog Neuropsychopharmacol Biol Psychiatry 32: 1793-1797, 2008.

27. Quincozes-Santos A, Abib RT, Leite MC, et al: Effect of the atypical neuroleptic risperidone on morphology and S100B secretion in C6 astroglial lineage cells. Mol Cell Biochem 314: 59-63, 2008.

28. Wu J, Chien CC, Yang LY, et al: Vitamin K3-2,3-epoxide induction of apoptosis with activation of ROS-dependent ERK and JNK protein phosphorylation in human glioma cells. Chem Biol Interact 193: 3-11, 2011.

29. Hoenicka J, Quiñones-Lombraña A, España-Serrano L, et al: The ANKK1 gene associated with addictions is expressed in astroglial cells and upregulated by apomorphine. Biol Psychiatry 67: 3-11, 2010.

30. Alentorn A,Marie Y, Carpentier C, et al: Prevalence, clinico-pathological value, and co-occurrence of PDGFRA abnormalities in diffuse gliomas. Neuro Oncol 14: 1393-1403, 2012.

31. Westermark B: Glioblastoma - a moving target. Ups J Med Sci 117: 251-256, 2012.

32. Puget S, Philippe C, Bax DA, et al: Mesenchymal transition and PDGFRA amplification/mutation are key distinct oncogenic events in pediatric diffuse intrinsic pontine gliomas. PLoS One 7: e30313, 2012.

33. Feng H, Hu B, Liu KW, et al: Activation of Rac1 by Src-dependent phosphorylation of Dock180(Y1811) mediates PDGFR $\alpha$-stimulated glioma tumorigenesis in mice and humans. J Clin Invest 121: 4670-4684, 2011.

34. Yang S, Wang K, Qian C, et al: A predicted miR-27a-mediated network identifies a signature of glioma. Oncol Rep 28: 1249-1256, 2012.

35. Buckman TD, Sutphin MS, and Mitrovic B: Oxidative stress in a clonal cell line of neuronal origin: effects of antioxidant enzyme modulation. J Neurochem 60: 2046-2058, 1993. 